\title{
Silicon-on-Insulator Tunable Wavelength Router with Minimized Polarization Dependent Loss
}

\author{
Robert Halir ${ }^{1}$, Diedrik Vermeulen ${ }^{2}$, Günther Roelkens ${ }^{2}$ \\ 1. Departamento Ingeniería de Comunicaciones, ETSI Telecomunicación, Universidad de Málaga, 29071 Málaga, Spain \\ 2. Department of Information Technology (INTEC), Ghent University-IMEC, B-9000 Gent, Belgium \\ robert.halir@ic.uma.es; diedrik.vermeulen@intec.ugent.be; gunther.roelkens@intec.ugent.be
}

\begin{abstract}
We demonstrate a novel polarization diversity scheme based on 2D grating couplers and a simple phase shifter to achieve polarization dependent loss as low as $0.15 \mathrm{~dB}$ in an SOI wavelength router

(C2010 Optical Society of America

OCIS codes: (060.4265) Networks, wavelength routing; (230.5440) Polarization-selective devices
\end{abstract}

\section{Introduction}

Fiber to the home (FTTH) applications demand cost-effective network components, including wavelength filters, power splitters and wavelength routers. Silicon-on-insulator is an attractive technology for the development of such devices, not only because it enables the design of extremely compact devices, but also because CMOS compatible mass-production is readily available [1]. However, such silicon-wire components exhibit a strong polarization dependence, which has to be overcome using polarization diversity configurations [2]. Here, we present a compact silicon-on-insulator wavelength router, capable of dynamic wavelength allocation and sharing between different users, which employs a novel polarization diversity approach to minimize polarization dependence loss at the transmission wavelengths.

\section{Tunable wavelength router design}

Fig. 1 shows a simple schematic of a flexible passive optical network, or FlexPON. In this example a total of eight wavelengths are used for communication between the central office and the four home users. Downstream data is transmitted on the wavelengths $\lambda_{d, 1 \ldots 4}$. The central office also sends the continuous wave wavelengths $\lambda_{c, 1 \ldots 4}$ which are modulated at the user end to transmit upstream data. The role of the wavelength router is to dynamically assign and share pairs of downstream and continuous wave wavelengths among the home users. In Fig. 1, users 2 and 3 each have a dedicated pair of wavelengths, whereas users 1 and 4 are sharing the same pair. If user 4 requests more bandwidth, the router can allocate a dedicated wavelength for this user.

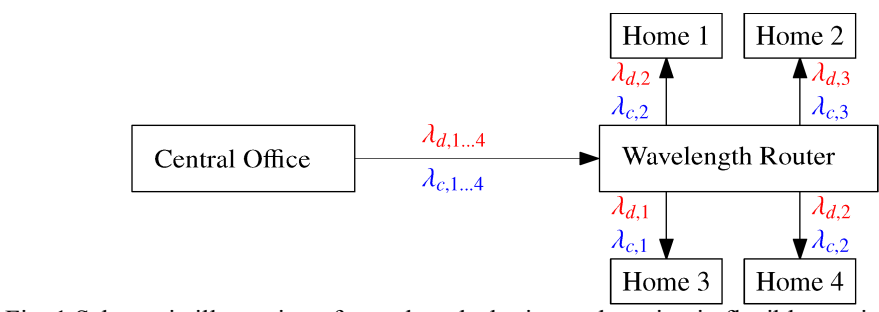

Fig. 1 Schematic illustration of wavelength sharing and routing in flexible passive optical network.

The design of the wavelength router is depicted in Fig. 2(a). Light is coupled into the chip on the left hand-side via a two dimensional grating coupler [3,4], which transforms the two orthogonal polarizations of the input fibers into TE polarization in each arm of the polarization diversity circuit. In order to allow for wavelength sharing, the signal is then divided with a $1 \times 4$ power splitter. The function of the waveguide heaters is related to the polarization dependent loss control technique and will be discussed later. Wavelength routing is performed at each output using thermally tunable ring resonators, and light is coupled back into the output fibers with identical two dimensional grating couplers. 


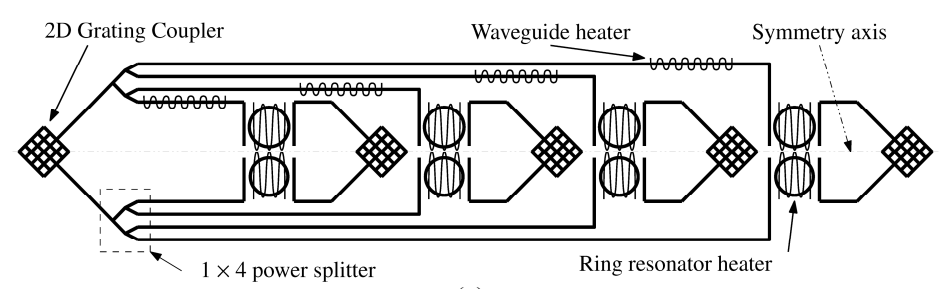

(a)

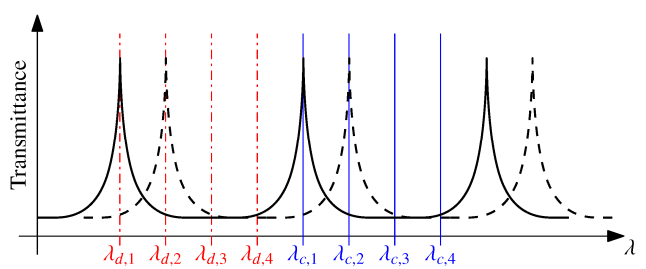

(b)

Fig. 2 (a) Design of the wavelength router. (b) Operation principle of ring resonators for wavelength routing.

The routing principle is illustrated in Fig. 2(b). The ring resonators have to be designed such that their free spectral range (FSR) matches the separation between the downstream and continuous wavelength of each pair. To route the pair $\lambda_{d, 1}, \lambda_{c, 1}$ the rings at the desired output are tuned to align their transmission maxima with these wavelengths while suppressing the other carriers (solid transmittance curve in Fig. 2(b)). By shifting the transmittance curve through thermal tuning, another pair of wavelengths can be selected (dashed curve in Fig. 2(b)). Polarization dependent loss (PDL) is thus critical at the transmission peaks, but is less important for the suppressed wavelength if the attenuation is large enough.

The fabricated device is based on completely etched, $220 \mathrm{~nm}$ thick and $450 \mathrm{~nm}$ wide single-mode Si-wire waveguides. The ring resonators were designed to have a FSR of $4.5 \mathrm{~nm}$, and use a racetrack configuration with a $4.5 \mu \mathrm{m}$ long coupling section where the gap between the waveguides is $180 \mathrm{~nm}$. The $1 \mathrm{x} 4$ splitter was implemented with a simple concatenation of 1x2 multimode interference couplers (MMI). A second etch depth of 70nm is used for the waveguide gratings, which consist of a 19x19 array of circular holes with a diameter of $400 \mathrm{~nm}$ and $605 \mathrm{~nm}$ pitch.

The grating couplers are designed for near vertical coupling $\left(\sim 10^{\circ}\right.$ with respect to the grating normal), in order to avoid strong back reflections that would arise from perfectly vertical second-order Bragg coupling. In order to preserve symmetry, the fiber has to be tilted along the diagonal of the grating, i.e., along the symmetry axis of the device shown in Fig. 2(a). The fiber polarization state that is oriented along this axis, and which we denote by $\mathrm{P}_{1}$, is then tilted slightly out of the plane of the grating, whereas the orthogonal polarization state $\left(\mathrm{P}_{2}\right)$ lays in the grating plane. This causes polarization states $\mathrm{P}_{1}$ and $\mathrm{P}_{2}$ to couple with different efficiencies, giving rise PDL in both the input and output gratings. However, from symmetry considerations it is clear that while polarization state $\mathrm{P}_{1}$ couples symmetrically, i.e. in phase, into the upper and lower arms of the polarization diversity circuit, polarization state P2 couples antisymmetrically, i.e. with a $180^{\circ}$ phase difference. Hence, by introducing a $180^{\circ}$ phase shift in one of the arms, $\mathrm{P}_{1}$ couples out as $\mathrm{P}_{2}$, and vice versa, so that each input polarization state experiences both coupling efficiencies (one at the input and the other one at the output), and PDL is minimized. The purpose of the waveguide heaters in Fig. 2(a) is precisely to produce this $180^{\circ}$ differential phase shift between the polarization diversity arms.

\section{Device fabrication and measurement}

The device was fabricated with deep UV lithography and a two step etch process on a commercial SOI wafer, and cladded with DVS-BCB. Titanium heaters were then defined on top of the rings, to allow for both matching of the two rings in each pair, as well as wavelength routing. The $180^{\circ}$ phase shifters in one of the arms of the polarization diversity circuit were also implemented with these titanium heaters.

A tunable laser source was used to record the transmission spectrum of the device, which is shown in Fig. 3. The insertion loss of around $22 \mathrm{~dB}$ is mainly attributed to the limited efficiency of the $2 \mathrm{D}$ grating couplers, which exhibit approximately $7 \mathrm{~dB}$ of loss each, as well as the $1 \times 4$ power splitter. A silicon overlay, as demonstrated in [5], can be used to improve the efficiency of the grating couplers. The rings exhibit the desired FSR, but their extinction ratio still needs be optimized. As shown in Fig. 3, the tuning characteristics required for wavelength routing were readily achievable by simultaneously heating the pair of rings in the polarization diversity configuration.

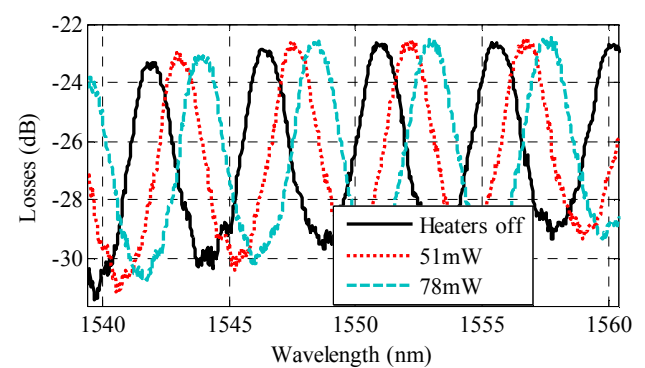

Fig. 3 Simultaneous tuning of the response of both rings in polarization diversity configuration for wavelength routing. 
PDL was measured at the transmission peak wavelengths using the Fiberpro "PL 2000" PDL meter. As shown in Fig. 4, when the $180^{\circ}$ phase shifter was inactive, around 1 to $2 \mathrm{~dB}$ of PDL were measured, which are mainly caused by the polarization dependence of the two dimensional grating couplers. Minimization of PDL using the $180^{\circ}$ phase shift described above is demonstrated in Fig. 4 for the transmission peaks at $1556.5 \mathrm{~nm}$ and 1560nm. Fig. 4(a) shows that by heating the waveguide with about $12 \mathrm{~mW}$ of power, and the ring heaters turned off, PDL can effectively be reduced to about $0.5 \mathrm{~dB}$. Tuning one of the ring heaters, with the waveguide heater turned off, does not yield significant improvement of PDL, indicating that the rings are closely matched. However, due to slight thermal crosstalk between the waveguide heaters and the ring heaters, they have to be fine-tuned to minimize PDL. When $6 \mathrm{~mW}$ of power are supplied to one of the ring heaters, and approximately $18 \mathrm{~mW}$ to the waveguide phase shifter, PDL is reduced to only $0.15 \mathrm{~dB}$. At $\lambda=1560 \mathrm{~nm}$ (Fig. $4(\mathrm{~b})$ ), a similarly drastic reduction in PDL is achieved, although with slightly different optimum heater powers. Similar results are obtained at all the transmission peaks, and, more importantly, by choosing intermediate heating powers, it is possible to reduced PDL at two consecutive transmission peaks to well below $0.5 \mathrm{~dB}$, as required for routing of the downstream and continuous wave wavelengths.
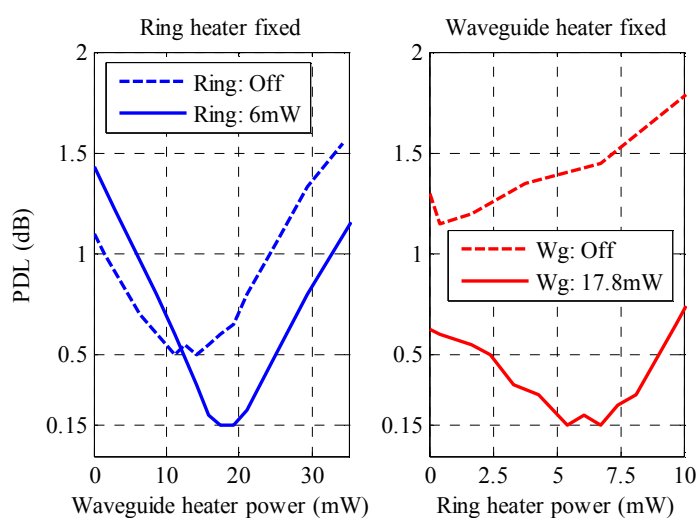

(a)
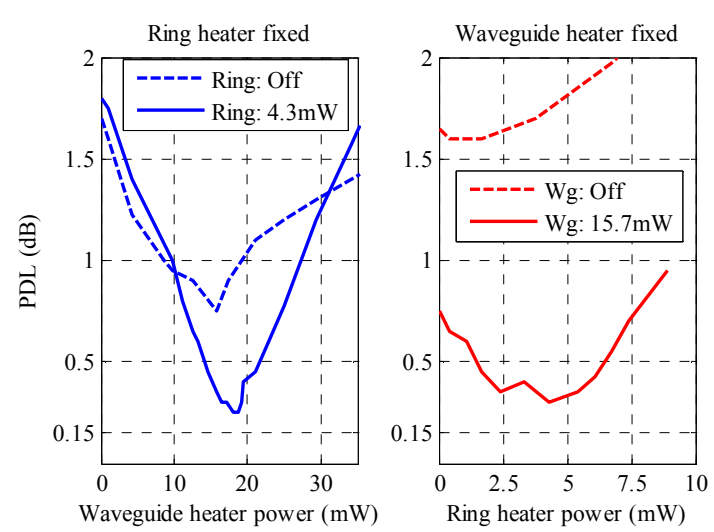

(b)

Fig. 4 Polarization dependent loss control by heating of one of the waveguide arms and re-tuning of the rings. (a) At $\lambda=1556.5 \mathrm{~nm},(\mathrm{~b})$ at $\lambda=1560 \mathrm{~nm}$

\section{Conclusions}

We have presented a tunable wavelength router that employs thermally adjusted ring resonators to dynamically assign and share wavelengths among a four users. The device simultaneously routes a data wavelength and an unmodulated carrier (for user modulated upstream data) to each user. The PDL at the transmission wavelengths was successfully reduced to well below $0.5 \mathrm{~dB}$ using a novel polarization diversity scheme with two dimensional grating fiber couplers and $180^{\circ}$ phase shifter.

\section{Acknowledgements}

This work was carried out in the framework of the Dutch Smartmix-Memphis project. Robert Halir acknowledges the Spanish Ministerio de Educación for the FPU scholarship with reference AP-2006-03355. D. Vermeulen thanks the Institute for the Promotion of Innovation by Science and Technology in Flanders (IWT) for a grant. G. Roelkens acknowledges the Fund for Scientific Research Vlaanderen (FWO) for a postdoctoral grant.

\section{References}

[1] Soref, R., "The Past, Present, and Future of Silicon Photonics", IEEE J. Sel. Top. Quantum Electron., 2006, 12, 1678-1687

[2] H. Fukuda, K. Yamada, T. Tsuchizawa, T. Watanabe, H. Shinojima, and S. Itabashi, "Silicon photonic circuit with polarization diversity," Opt. Express, vol. 16, pp. 4872-4880, 2008

[3] D. Taillaert, H. Chong, P. Borel, L. Frandsen, R. De La Rue, and R. Baets, "A compact two-dimensional grating coupler used as a polarization splitter," IEEE Photonics Technol. Lett., vol. 15, no. 9, pp. 1249-1251, 2003.

[4] F. Van Laere, T. Stomeo, D. Taillaert, G. Roelkens, D. Van Thourhout, T. Krauss, and R. Baets, "Efficient Polarization Diversity Grating Couplers in Bonded InP-Membrane,” IEEE Photonics Technol. Lett., vol. 20, no. 4, 2008.

[5] G. Roelkens, D. Vermeulen, D. Van Thourhout, R. Baets, S. Brision, P. Lyan, P. Gautier, and J. Fédéli, "High efficiency diffractive grating couplers for interfacing a single mode optical fiber with a nanophotonic silicon-on-insulator waveguide circuit," Appl. Phys. Lett, vol. 92, pp. $131101-131103,2008$. 